\title{
The efficacy and safety of apatinib in Ewing's sarcoma: a retrospective analysis in one institution
}

This article was published in the following Dove Press journal: Cancer Management and Research

\section{Yitian Wang* \\ Li Min* \\ Yong Zhou \\ Yi Luo \\ Hong Duan \\ Chongqi Tu}

Department of Orthopedics, West China Hospital, Sichuan University, Chengdu, Sichuan 61004I, People's Republic of China

*These authors contributed equally to this work

Correspondence: Chongqi Tu

Department of Orthopedics, West China Hospital, Sichuan University, No. 37

Guoxuexiang, Chengdu, Sichuan 61004I, People's Republic of China

Tel +86 I39 80095430

Fax +86 2885582944

Email tuchongqibone@hotmail.com
Background: Ewing's sarcoma (ES) is a highly aggressive and metastatic neoplasm occurring mainly in children and young adults. The standard treatment of localized ES requires a combination of surgery, chemotherapy, and radiotherapy. Although the 5-year survival rate for local ES has improved, the survival rate and prognosis are still very poor for metastatic or recurrent ES patients. The aim of this study was to investigate the efficacy and safety of apatinib, a specific vascular endothelial growth factor receptor 2 inhibitor, in ES patients.

Methods: This retrospective analysis involved eleven patients with ES not amenable to curative treatment. All patients suffered poor responses to two cycles of chemotherapy (vincristine, doxorubicin, and cyclophosphamide). Apatinib $500 \mathrm{mg}$ (or $250 \mathrm{mg}$ ) was given daily. Tumor responses were assessed according to the Response Evaluation Criteria in Solid Tumors 1.1. Survival analysis was performed by the Kaplan-Meier test. The safety profile was also recorded. Results: The mean age of the patients was 18 (range, 10-31) years. The 12-month overall survival and progression-free survival rates were $90 \%$ and $72 \%$, respectively. Four patients achieved partial response, and four patients achieved stable disease, with objective response rate of $40 \%$. The median follow-up in our study was 16 months (range, 3-26 months). The most common adverse events included hand-foot skin reaction $(n=5 ; 45 \%)$, oral ulcers $(n=4 ; 36 \%)$, and gastrointestinal discomfort $(\mathrm{n}=4 ; 36 \%)$.

Conclusion: Apatinib may provide as second- or first-line treatment options for ES patients, particularly in chemoresistant cases. Further studies with more cases and longer follow-up will be necessary to determine the clinical efficacy and safety of apatinib in ES patients.

Keywords: Ewing's sarcoma, apatinib, vascular endothelial growth factor, efficacy, safety

\section{Introduction}

Ewing sarcoma family tumors (ESFTs) are a group of aggressive neoplasms of the bone and soft tissue, characterized histologically by small, round, blue cells. ESFTs mainly occur in adolescents and young adults, between ages 10 and 20 years, as primitive neuroectodermal tumor. Specifically, they are malignant small-cell tumors of the thoracopulmonary region and classic Ewing sarcomas of the bone and soft tissue. ${ }^{1,2}$ Ewing's sarcoma (ES) is characterized by chromosomal translocation involving the Ewing sarcoma breakpoint region 1 (EWSR1) gene, and $85 \%$ of cases are associated with translocations of EWSR1 on chromosome 22 to chromosome 11. ${ }^{2,3}$

Nearly $27 \%$ of ES patients are found to have metastatic lesions at diagnosis, and the remaining patients have localized cancer. ${ }^{4}$ A multidisciplinary approach including risk-adapted chemotherapy and local therapy (surgery and radiation therapy) has 
significantly increased the survival of patients with localized disease. ${ }^{5}$ However, the prognosis of advanced ES patients is still grave, with 5 -year survival rates of only $20 \%-30 \%{ }^{6,7}$ According to the Clinical Practice Guideline of the National Comprehensive Cancer Network, the first-line treatment with systemic therapy is recommended, including vincristine, doxorubicin, cyclophosphamide, ifosfamide, and etoposide. Nevertheless, the second-line therapy of ES depends on previous completion of adjuvant therapy and first-line treatment, as well as patient's tolerance.

Several second-line chemotherapy regimens have been recommended for refractory or recurrent ES, ${ }^{8,9}$ cancers that are typically refractory to conventional chemotherapy and irradiation. ${ }^{10}$ In addition, there are still no randomized studies comparing different regimens for the second-line chemotherapy treatments. Moreover, the best second-line regimens have not been identified, and further investigation will be required to identify them.

Apatinib is a novel receptor tyrosine kinase inhibitor that selectively competes for the vascular endothelial growth factor receptor 2 (VEGFR-2) ATP-binding site, blocking downstream signaling, and inhibiting tumor angiogenesis. ${ }^{11,12}$ Apatinib has been reported to improve progression-free survival (PFS), and consequently overall survival (OS), in patients with advanced gastric cancer. ${ }^{13}$ It is thought to be a potentially useful agent to expand the therapeutic options in various sarcomas, including angiosarcoma, malignant fibrous histiocytoma, and myxoid/round cell liposarcoma. ${ }^{14,15}$

To our best knowledge, there are no prior reports on the effects of apatinib on ES, and therefore, the aim of this study was to investigate the efficacy and safety of apatinib, a specific VEGFR-2 inhibitor, in patients with these cancers. We conducted a retrospective cohort study to investigate the association of antiangiogenesis-related adverse events (AEs) with clinical outcomes in ES patients and to report the data from a total of eleven of these patients treated with apatinib. Our report describes the usage, safety, and efficacy of apatinib in ES patients, who were being treated in the Department of Orthopedics at West China Hospital.

\section{Patients and methods Eligibility criteria}

The study was conducted retrospectively on patients treated between February 1, 2016, and January 1, 2018. The inclusion criteria included 1) histologically proven ES, 2) initial treatment in the Department of Orthopedics of West China Hospital, 3) tumors that were not amenable to curative treatment (with unresectable lesions or distant metastasis), 4) measur- able lesions according to Response Evaluation Criteria for Solid Tumors (RECIST), ${ }^{16}$ 5) an Eastern Cooperative Oncology Group performance status 0 or 1 , and 6) chemotherapy rejection or at least two prior cycles of chemotherapy, including VAC (vincristine, doxorubicin, and cyclophosphamide) or VAIE (vincristine, doxorubicin, and cyclophosphamide alternating with ifosfamide and etoposide).

\section{Treatment methods}

Because these patients had not responded to conventional chemotherapy, apatinib was administered at a dose of 500 $\mathrm{mg}$ once daily. For patients younger than 10 years, we used $250 \mathrm{mg}$ once daily. ${ }^{17}$ One treatment cycle was 28 days long. One dose reduction (to $250 \mathrm{mg}$ ) or interruptions of therapy for drug-related toxicity was allowed. Dose-limiting toxicity was defined as possibly or definitely drug-related grades 3-4 toxic responses. This study was performed according to the principles embodied in the Declaration of Helsinki and the Institutional Review Board of Sichuan University West China Hospital. Written informed consent was obtained from all patients when they began treatment for apatinib. The study protocol followed all appropriate guidelines according to the Declaration of Helsinki.

\section{Evaluation of efficacy and safety}

In this retrospective study, we were mainly concerned with documenting the objective response rate (ORR), including complete response and partial response (PR) cases, as well as the PFS for each apatinib protocol according to RECIST 1.1. Moreover, OS and toxicities encountered in the study were also recorded. Treatment efficacy was evaluated by computed tomography (CT) or magnetic resonance imaging. The safety evaluation was based on the frequency and severity of events assessed according to the Common Terminology Criteria for Adverse Events. ${ }^{18}$ Kaplan-Meier survival curves were used for PFS estimation. Data analyses were performed using SPSS 20.0 software (IBM Corporation, Armonk, NY, USA).

\section{Results}

\section{Patients' characteristics}

From February 1, 2016, to January 1, 2018, eleven consecutive advanced ES patients were enrolled. All pathologic diagnoses were confirmed at West China Hospital. Patients' clinical characteristics at entry to the study are summarized in Table 1. One patient was lost to follow-up, and one patient stopped using apatinib because of toxicity. Median followup time was 15.8 months (range, 3.0-26.0 months). Before treatment with apatinib, first line of prior chemotherapy was 
Table I Patients' characteristics

\begin{tabular}{|c|c|}
\hline Characteristics & Number of patients \\
\hline \multicolumn{2}{|l|}{ Sex } \\
\hline Male & $6(54.5)$ \\
\hline Female & $5(45.5)$ \\
\hline \multicolumn{2}{|l|}{ Age (years) } \\
\hline Median & $18(10-3 \mid)$ \\
\hline $10-20$ & $6(54.5)$ \\
\hline $20-30$ & $4(36.4)$ \\
\hline$>30$ & I (9) \\
\hline \multicolumn{2}{|l|}{ Metastatic site } \\
\hline No metastasis & $4(36.4)$ \\
\hline Lung only & $4(36.4)$ \\
\hline Bone only & $I(9.1)$ \\
\hline Others $^{\mathrm{a}}$ & $2(18.1)$ \\
\hline \multicolumn{2}{|c|}{ Number of previous treatment lines } \\
\hline 1 & $9(81.8)$ \\
\hline 2 & $0(0)$ \\
\hline$>2$ & $I(9.1)$ \\
\hline Others $^{\mathrm{b}}$ & $I(9.1)$ \\
\hline
\end{tabular}

Notes: Data are represented as $\mathrm{n}(\%)$ and median (range). ${ }^{\mathrm{a}}$ Others including lung and spine; $b$ other means the patient can not provide the chemotherapy regimens

administrated for at least two cycles. Seven patients (63.6\%) had metastasis at initial presentation (including lung, spine, and bone metastasis).

\section{Treatment methods}

Among eleven patients, five (45.4\%) patients received chemotherapy, local surgery, and then apatinib. Another five $(45.4 \%)$ patients received apatinib and chemotherapy in combination, and stable disease (SD) was achieved. The last one $(9.2 \%)$ patient received only apatinib, because chemotherapy was rejected after a lung metastasis was found at the initial diagnosis (Table 2).

All eleven patients were evaluated by physicians in our clinic on a monthly basis and with at least chest $\mathrm{CT}$ and imaging of tumor lesions at other sites.

\section{Efficacy}

By the last scheduled visit (Table 3), one patient was lost to follow-up and one patient died of lung metastasis. The median PFS was 16.0 months $(95 \% \mathrm{CI})$. Four $(40 \%)$ of ten patients had PRs (Figure 1), and four (40\%) had SD (Table 4). Two (20\%) patients developed progressive disease (PD). The 6- and 12 -month OS rates were $90.0 \%$ and $78.8 \%$, respectively. Moreover, the 6- and 12-month PFS rates were $81.8 \%$ and $71.6 \%$, respectively (Figures 2 and 3). The ORR was 40\% (4/10) for ES.

\section{Safety and toxicity}

The treatment was interrupted in one $(10 \%)$ patient because of hand-foot syndrome. Two patients (15- and 19-year-old
Table 2 Different treatment combinations and response

\begin{tabular}{|l|l|l|}
\hline Target therapy & n (\%) & Best response $^{\mathbf{a}}$ \\
\hline Apatinib alone & I (9.I) & PD \\
\hline Chemotherapy+apatinib $^{\mathrm{b}}$ & $5(45.5)$ & PR \\
\hline Chemotherapy+surgery+apatinib $^{c}$ & $5(45.5)$ & PR \\
\hline
\end{tabular}

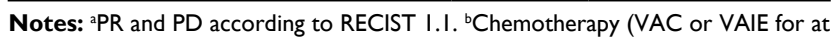
least two cycles) was administered with apatinib. ${ }^{c}$ Chemotherapy and surgery were administered with apatinib.

Abbreviations: PD, progressive disease; PR, partial response; RECIST, Response Evaluation Criteria in Solid Tumors; SD, stable disease; VAC, vincristine, doxorubicin, and cyclophosphamide; VAIE, vincristine, doxorubicin, and cyclophosphamide alternating with ifosfamide and etoposide.

males) died of lung metastasis because of respiratory failure. Toxicities encountered in the study are listed in Table 5. Most adverse reactions were mild (grades 1-2) and easily controlled. However, some more serious adverse reactions occurred and were of variable severity. The only grade 3 reaction was hand-foot syndrome.

\section{Discussion}

ES is aggressive with high metastatic tendency and can develop resistance to conventional chemotherapy. ${ }^{19}$ The mechanisms by which ES become resistant to chemotherapy are varied and can involve inherent resistance of the cancer stem cells, drug metabolizing enzymes, modulation of cellular signal transduction pathways, and tumor heterogeneity. ${ }^{19}$

Several phase I and II clinical trials have reported investigational therapies for ES, including insulin growth factorreceptor antibody, mammalian target of rapamycin inhibition, poly ADP-ribose polymerase inhibition, telomerase inhibition, Akt, nuclear factor $\kappa \mathrm{B}, B R A F$, and vascular endothelial growth factor (VEGF). ${ }^{20-22}$ Owing to obvious side effects and translation failure of these observations into clinical strategies, multiple parallel pathways and cellular processes will need further investigation.

Among these possible therapeutic targets, VEGF has been highlighted in ES cell lines. Inhibition or blockade of VEGF can result in the suppression of ES tumor growth. ${ }^{23}$ There also appears to be an association of VEGF expression with poor prognosis of ES patients. ${ }^{24-28}$ VEGF has an important role in tumor angiogenesis and vasculogenesis. Although there are many subtypes of VEGF, VEGFR-2 is the key mediator of the recognized VEGF-induced phenotypes. ${ }^{29}$ Apatinib, a specific VEGFR-2 inhibitor, was licensed by the China Food and Drug Administration for the treatment of advanced/metastatic gastric cancer and adenocarcinomas of the gastroesophageal junction in 2014. Its anticancer effects have been reported in a broad range of malignancies such as osteosarcoma, rhabdomyosarcoma, synovial sarcoma, and alveolar soft part sarcoma. ${ }^{17,30}$ 


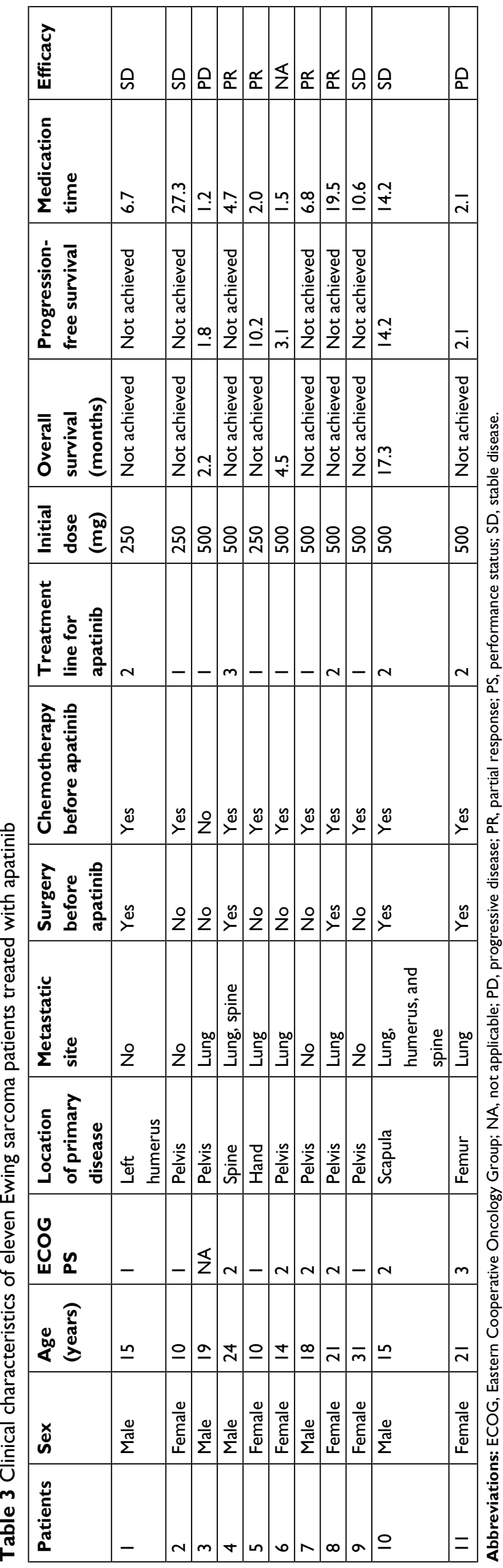

Table 4 Patients' clinical evaluations

\begin{tabular}{|l|l|}
\hline Clinical evaluations & Number of patients \\
\hline $\mathrm{n}$ (missing) & $\mathrm{I}(\mathrm{I})$ \\
\hline $\mathrm{CR}$ & $0(0.00)$ \\
\hline $\mathrm{PR}$ & $4(40.00)$ \\
\hline $\mathrm{SD}$ & $4(40.00)$ \\
\hline PD & $2(20.00)$ \\
\hline ORR $(95 \% \mathrm{Cl})$ & $40.00(12.16-73.76)$ \\
\hline DCR $(95 \% \mathrm{Cl})$ & $80.00(44.39-97.48)$ \\
\hline
\end{tabular}

Note: Data are represented as $\mathrm{n}(\%)$ and median (range).

Abbreviations: CR, complete response; DCR, disease control rate; ORR, objective response rate; PD, progressive disease; PR, partial response; SD, stable disease.

Previous studies have evaluated the response of advanced bone and soft tissue sarcomas to apatinib. Zhu et al ${ }^{30}$ reported an ORR of $33.3 \%$ when apatinib was administered as a second-line or further-line treatment. Xie et al reported, as a second-line or further-line treatment, the ORR was $40.9 \%(9 / 22)$ for osteosarcoma, $70 \%(7 / 10)$ for ES, $100 \%$ (3/3 cases) for chondrosarcoma, and 71.4\% (15/21) for soft tissue sarcoma. ${ }^{17}$ Thus, apatinib was a third-line treatment option for one ES patient in our study, with the result encouraging as a PR. In fact, apatinib has been reported as a first-line treatment for off-label use. ${ }^{31,32}$ Therefore, we attempted to use apatinib as a second- or first-line treatment option for ES patients, particularly in cases resistant to chemotherapy and five of the ten ES patients took apatinib together with chemotherapy. Apatinib also was administered as a first-line treatment option for advanced ES patients in this study. Median follow-up in our study was 16.0 months, and one patient (10\%) achieved a PD, two (20\%) achieved SD, and two (20\%) achieved PR. For four patients (40\%) used apatinib as second-line treatment, one $(10 \%)$ achieved a PD, two (20\%) achieved a SD, and one (10\%) developed a PR. In our study, the ORR with apatinib was $40 \%(4 / 10)$ in ES. Although Xie et al reported that their objective ORR was $70 \%(7 / 10)$ for ES patients, and our ORR rate was $40 \%$ (4/10), their patients met endpoints in a short period. We have achieved 12-month OS and PFS rates of $90.0 \%$ and $71.6 \%$, respectively. As most patients are still assessed as having PR or SD, the duration of response of patients could not be evaluated. Apatinib was administered at a dose of $500 \mathrm{mg} /$ day for adolescents and adults and $250 \mathrm{mg} /$ day $^{30}$ for patients younger than 10 years, dosing that is lower than that used in the previous trials. Therefore, our ORR may have been limited by the lower drugs doses.

For the AEs, the most common toxicities are fatigue, hypertension, and hand-foot skin reaction (HFS) ${ }^{30}$ Importantly, only one patient had to stop using apatinib as a result 


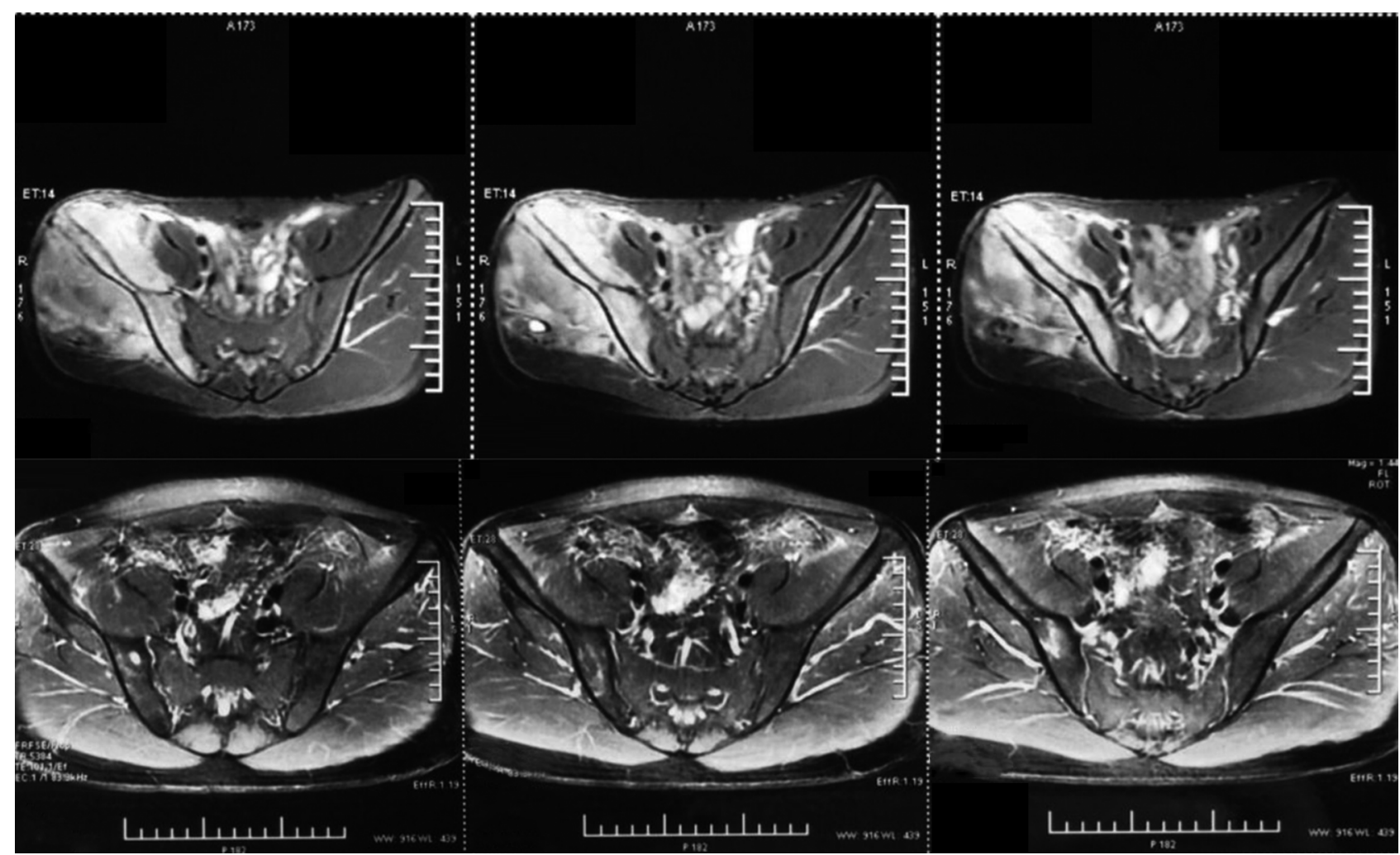

Figure I The patient with PR showing response to the treatment with apatinib.

Note: MRIs of the pelvis are shown before and after treatment.

Abbreviations: MRI, magnetic resonance imaging; PR, partial response.

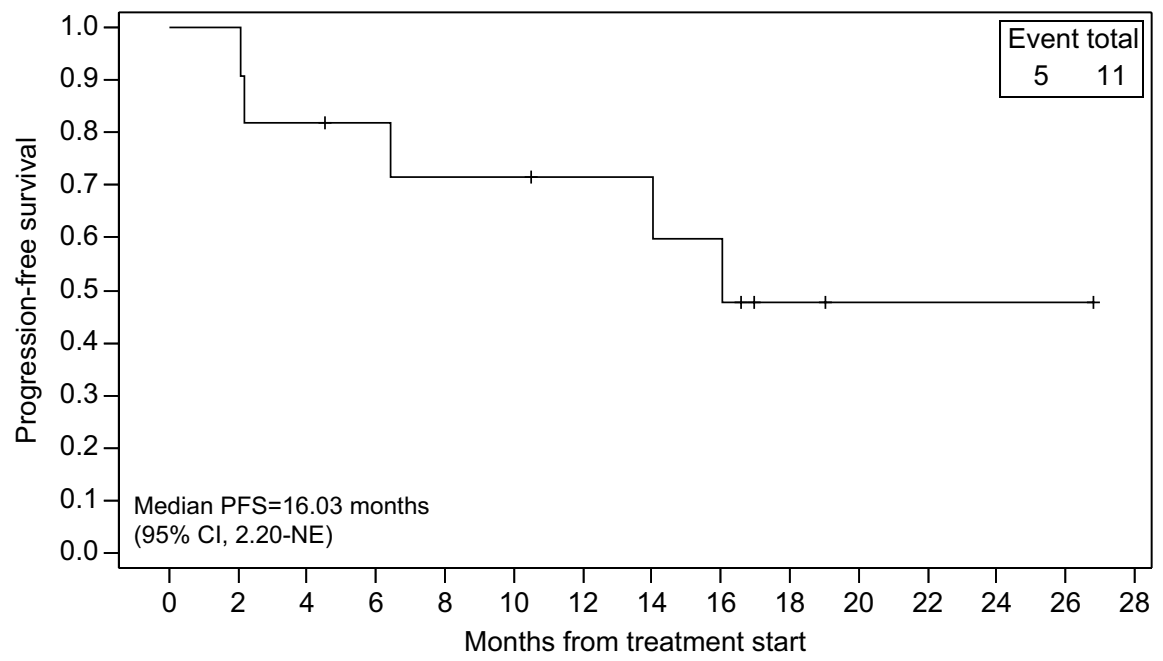

\begin{tabular}{|c|c|c|c|c|c|c|c|c|c|c|c|c|c|c|c|}
\hline & & & & & & 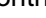 & . & . & 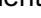 & & & & & & \\
\hline & & & & & & iens & & 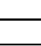 & $\mathrm{P}$ & bak & ty $c$ & urv & & & \\
\hline Number at risk & 11 & 11 & 9 & 8 & 7 & 7 & 6 & 6 & 5 & 2 & 1 & 1 & 1 & 1 & 0 \\
\hline
\end{tabular}

Figure 2 Kaplan-Meier estimates of PFS for all patients.

Abbreviation: PFS, progression-free survival.

of toxicity (HFS). Four patients had oral ulcers and weight loss. Five of our patients had treatment-related gastrointestinal discomfort, and one patient had delayed wound healing. None of our patients had hypertension. Interestingly, previous studies reported that patients with grade $3 / 4$ toxicities had a longer PFS than those without grade $3 / 4$ toxicities, especially for patients with hypertension and HFS. ${ }^{33}$ Similar results were found in our study. 


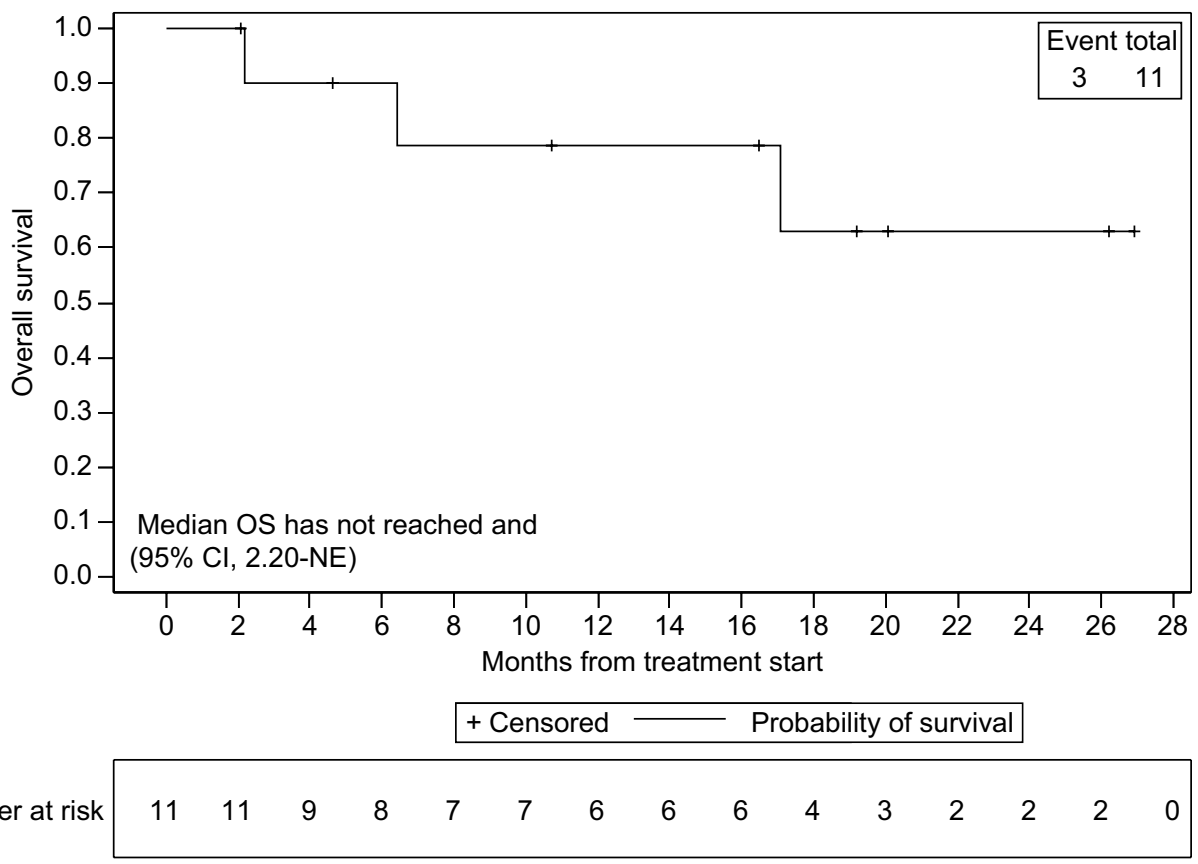

Figure 3 Kaplan-Meier estimates of OS for all patients.

Abbreviation: OS, overall survival.

Table 5 Adverse events

\begin{tabular}{|l|l|l|l|l|}
\hline \multirow{2}{*}{ Adverse events } & \multirow{2}{*}{ Total, n (\%) } & \multicolumn{3}{|l|}{ Grade } \\
\cline { 3 - 5 } & & I & $\mathbf{2}$ & $3-4$ \\
\hline Hand-foot skin reaction & 5 & 2 & 2 & $\mathrm{I}$ \\
\hline Anorexia & 2 & 2 & 0 & 0 \\
\hline Proteinuria & $\mathrm{I}$ & 0 & $\mathrm{I}$ & 0 \\
\hline Hair hypopigmentation & 2 & 0 & 2 & 0 \\
\hline Wound-healing problems & $\mathrm{I}$ & 0 & $\mathrm{I}$ & 0 \\
\hline Oral ulcers & 4 & $\mathrm{I}$ & 3 & 0 \\
\hline Gastrointestinal discomfort ${ }^{\mathrm{a}}$ & 4 & 2 & 2 & 0 \\
\hline Thrombocytopenia & $\mathrm{I}$ & $\mathrm{I}$ & 0 & 0 \\
\hline Epistaxis & $\mathrm{I}$ & 0 & $\mathrm{I}$ & 0 \\
\hline Elevated aminotransferase or bilirubin & $\mathrm{I}$ & $\mathrm{I}$ & 0 & 0 \\
\hline
\end{tabular}

Note: ${ }^{a}$ Gastrointestinal discomfort means nausea/vomiting.

We recognize that our study has some limitations. First, as a retrospective study, most patients had combined chemotherapy. Therefore, it is not strictly correct to draw comparisons with the used of apatinib and other drugs. Second, the inclusion criteria utilized were not as rigorous as those employed in formal prospective trials. Third, because only eleven ES patients were enrolled in our study, we had insufficient numbers to permit subset analyses and limited statistical power. Fourth, the dose of $250 \mathrm{mg}$ apatinib adopted in this study for some patients was not widely recommended.

\section{Conclusion}

This study provides preliminary evidence of efficacy of apatinib in patients with advanced ES. Apatinib may prove to be a second-line or even a first-line treatment option for advanced ES patients, particularly in cases resistant to chemotherapy. Further studies with more patients and longer follow-up times will be necessary to fully determine the clinical efficacy of apatinib in ES. Indeed, it is likely that formal multicenter randomized clinical trials will be required to fully establish the effects of apatinib on ES patients.

\section{Ethical statement}

To preserve patient confidentiality and privacy, patient data have been deidentified before analysis. This retrospective study was performed based on the data from anonymized patients who received apatinib treatment between February 2016 and January 2018. Because of the nature of retrospective design and patient anonymization, the ethical board of Sichuan University West China Hospital approved the retrospective study and also determined that informed consent was not required.

\section{Acknowledgments}

This work was supported, in part, by National Natural Science Foundation of China (81702664). This abstract was selected for online publication presenting interim findings only, at the 
2018 ASCO Annual Meeting, taking place on June 1-5, 2018, at the McCormick Place Convention Center in Chicago, Illinois (Abstract Submission ID: 211471; Password: 210783). The abstract was released by ASCO on May 16, 2018, at 5:00 PM EDT on meetinglibrary.asco.org.

\section{Disclosure}

The authors report no conflicts of interest in this work.

\section{References}

1. Rosen G, Wollner N, Tan C, et al. Proceedings: disease-free survival in children with Ewing's sarcoma treated with radiation therapy and adjuvant four-drug sequential chemotherapy. Cancer. 1974;33(2):384-393.

2. Burchill SA. Molecular abnormalities in Ewing's sarcoma. Expert Rev Anticancer Ther. 2008;8(10):1675-1687.

3. Jiang Y, Ludwig J, Janku F. Targeted therapies for advanced Ewing sarcoma family of tumors. Cancer Treat Rev. 2015;41(5):391-400.

4. Esiashvili N, Goodman M, Marcus RB. Changes in incidence and survival of Ewing sarcoma patients over the past 3 decades: Surveillance Epidemiology and End Results data. J Pediatr Hematol Oncol. 2008;30(6):425-430.

5. Krasin MJ, Davidoff AM, Rodriguez-Galindo C, et al. Definitive surgery and multiagent systemic therapy for patients with localized Ewing sarcoma family of tumors: local outcome and prognostic factors. Cancer. 2005;104(2):367-373.

6. Cotterill SJ, Ahrens S, Paulussen M, et al. Prognostic factors in Ewing's tumor of bone: analysis of 975 patients from the European Intergroup Cooperative Ewing's Sarcoma Study Group. J Clin Oncol. 2000;18(17):3108-3114.

7. van Maldegem AM, Benson C, Rutkowski P, et al. Etoposide and carbo- or cisplatin combination therapy in refractory or relapsed Ewing sarcoma: a large retrospective study. Pediatr Blood Cancer. 2015;62(1):40-44.

8. Fox E, Patel S, Wathen JK, et al. Phase II study of sequential gemcitabine followed by docetaxel for recurrent Ewing sarcoma, osteosarcoma, or unresectable or locally recurrent chondrosarcoma: results of Sarcoma Alliance for Research Through Collaboration Study 003. Oncologist. 2012;17(3):321.

9. Raciborska A, Bilska K, Drabko K, et al. Vincristine, irinotecan, and temozolomide in patients with relapsed and refractory Ewing sarcoma. Pediatr Blood Cancer. 2013;60(10):1621-1625.

10. Arpaci E, Yetisyigit T, Seker M, et al. Prognostic factors and clinical outcome of patients with Ewing's sarcoma family of tumors in adults: multicentric study of the Anatolian Society of Medical Oncology. Med Oncol. 2013;30(1):469.

11. Li J, Zhao X, Chen L, et al. Safety and pharmacokinetics of novel selective vascular endothelial growth factor receptor-2 inhibitor YN968D1 in patients with advanced malignancies. BMC Cancer. 2010;10:529.

12. Ding J, Chen X, Gao Z, et al. Metabolism and pharmacokinetics of novel selective vascular endothelial growth factor receptor-2 inhibitor apatinib in humans. Drug Metab Dispos. 2013;41(6):1195-1210.

13. Li J, Qin S, Xu J, et al. Randomized, double-blind, placebo-controlled phase III trial of apatinib in patients with chemotherapy-refractory advanced or metastatic adenocarcinoma of the stomach or gastroesophageal junction. J Clin Oncol. 2016;34(13):1448-1454.

14. Ji G, Hong L, Yang P. Successful treatment of angiosarcoma of the scalp with apatinib: a case report. Onco Targets Ther. 2016;9:4989-4992.
15. Ji G, Hong L, Yang P. Successful treatment of advanced malignant fibrous histiocytoma of the right forearm with apatinib: a case report. Onco Targets Ther. 2016;9:4989-4992.

16. Taylor PT, Haverstick D. Re: new guidelines to evaluate the response to treatment in solid tumors (ovarian cancer). $J$ Natl Cancer Inst. 2005;97(2):151.

17. Xie L, Guo W, Wang Y, Yan T, Ji T, Xu J. Apatinib for advanced sarcoma: results from multiple institutions' off-label use in China. BMC Cancer. 2018;18(1):396.

18. de Rojas T, Bautista FJ, Madero L, Moreno L. The first step to integrating adapted common terminology criteria for adverse events for children. J Clin Oncol. 2016;34(18):2196-2197.

19. Ahmed AA, Zia H, Wagner L. Therapy resistance mechanisms in Ewing's sarcoma family tumors. Cancer Chemother Pharmacol. 2014;73(4):657-663.

20. Vornicova O, Bar-Sela G. Investigational therapies for Ewing sarcoma: a search without a clear finding. Expert Opin Investig Drugs. 2016;25(6):679-686.

21. Arnaldez FI, Helman LJ. New strategies in Ewing sarcoma: lost in translation? Clin Cancer Res. 2014;20(12):3050-3056.

22. Naing A, Lorusso P, Fu S, et al. Insulin growth factor-receptor (IGF-1R) antibody cixutumumab combined with the mTOR inhibitor temsirolimus in patients with refractory Ewing's sarcoma family tumors. Clin Cancer Res. 2012;18(9):2625-2631.

23. Yudoh K, Kanamori M, Ohmori K, Yasuda T, Aoki M, Kimura T. Concentration of vascular endothelial growth factor in the tumour tissue as a prognostic factor of soft tissue sarcomas. Br J Cancer. 2001;84(12): 1610-1615.

24. Yudoh K, Kanamori M, Ohmori K, Yasuda T, Aoki M, Kimura T. Concentration of vascular endothelial growth factor in the tumour tissue as a prognostic factor of soft tissue sarcomas. Br J Cancer. 2001;84(12): 1610-1615.

25. Dalal S, Berry AM, Cullinane CJ, et al. Vascular endothelial growth factor: a therapeutic target for tumors of the Ewing's sarcoma family. Clin Cancer Res. 2005;11(6):2364-2378.

26. Zhou Z, Bolontrade MF, Reddy K, et al. Suppression of Ewing's sarcoma tumor growth, tumor vessel formation, and vasculogenesis following anti vascular endothelial growth factor receptor-2 therapy. Clin Cancer Res. 2007;13(16):4867-4873.

27. Ahmed AA, Sherman AK, Pawel BR. Expression of therapeutic targets in Ewing sarcoma family tumors. Hum Pathol. 2012;43(7): 1077-1083.

28. Kreuter M, Paulussen M, Boeckeler J, et al. Clinical significance of vascular endothelial growth factor-A expression in Ewing's sarcoma. Eur J Cancer. 2006;42(12):1904-1911.

29. Autiero M, Waltenberger J, Communi D, et al. Role of PlGF in the intra- and intermolecular cross talk between the VEGF receptors Flt1 and Flk1. Nat Med. 2003;9(7):936-943.

30. Zhu B, Li J, Xie Q, Diao L, Gai L, Yang W. Efficacy and safety of apatinib monotherapy in advanced bone and soft tissue sarcoma: an observational study. Cancer Biol Ther. 2018;19(3):198-204.

31. Wang Y, Bi M, Zhang H, Gao Z, Zhou H, Chang S. Apatinib in gastric carcinoma: a case report of partial response for first-line treatment in advanced disease. Asia Pac J Clin Oncol. 2017;13(5):e528-e530.

32. Dong M, Bi J, Liu X, Wang B, Wang J. Significant partial response of metastatic intra-abdominal and pelvic round cell liposarcoma to a small-molecule VEGFR-2 tyrosine kinase inhibitor apatinib: a case report. Medicine. 2016;95(31):e4368.

33. Li J, Wang L. Efficacy and safety of apatinib treatment for advanced esophageal squamous cell carcinoma. Onco Targets Ther. 2017;10:3965-3969. 


\section{Publish your work in this journal}

Cancer Management and Research is an international, peer-reviewed open access journal focusing on cancer research and the optimal use of preventative and integrated treatment interventions to achieve improved outcomes, enhanced survival and quality of life for the cancer patient. The manuscript management system is completely online and includes a very quick and fair peer-review system, which is all easy to use. Visit http://www.dovepress.com/testimonials.php to read real quotes from published authors.

Submit your manuscript here: https://www.dovepress.com/cancer-management-and-research-journal 